\title{
Effect of Air-powder Polishing on the Surface Topography of Orthodontic Stainless Steel Wires
}

\author{
Nabeel F Talic
}

\section{ABSTRACT}

Aim: The aim of this study was to examine the effect of in vivo air-powder polishing on the surface roughness and topography of aged orthodontic stainless steel arch wires.

Materials and methods: A total of 20 stainless steel arch wires were used in this study. Ten wires (experimental) were aged and exposed to air-powder polishing, and 10 wires asreceived from the manufacturer served as the controls. The experimental group of wires was aged in the patient's oral cavity for an average of $5.3 \pm 2.7$ months. An air-powder polishing system was used to remove external stains and plaque deposits. The powder was sodium bicarbonate. The surface topography measurements were performed using an optical profilometer. Following the profilometer analysis, a scanning electron microscope (SEM) was used to evaluate the experimental and control arch wires.

Results: The average roughness (Sa) of the experimental upper and lower surfaces exposed to air-powder polishing was significantly higher than the control arch wires $(p<0.01)$. The root mean square of the surface topography $(\mathrm{Sq})$ and the 10-point height of the surface topography (Sz) were significantly higher on the upper and lower surfaces of the experimental arch wires compared with the controls $(p<0.05$ and $<0.01$ respectively). The SEM photomicrographs of the experimental and control arch wires indicated that the experimental arch wires were pitted and filled with surface impurities.

Conclusion: An air-powder polishing system using sodium bicarbonate powder alters the surface topography and increases the surface roughness of aged orthodontic arch wires in vitro.

Clinical significance: The air-powder polishing system used along with scaling alters the surface roughness of orthodontic wire.

Keywords: Air-powder polishing, Optical profilometer, Orthodontic arch wires, Scanning electron microscope.

How to cite this article: Talic NF. Effect of Air-powder Polishing on the Surface Topography of Orthodontic Stainless Steel Wires. World J Dent 2017;8(4):262-266.

Source of support: Nil

Conflict of interest: None

Department of Pediatric Dentistry and Orthodontics, College of Dentistry, King Saud University, Riyadh, Kingdom of Saudi Arabia

Corresponding Author: Nabeel F Talic, Department of Pediatric Dentistry and Orthodontics, College of Dentistry, King Saud University, Riyadh, Kingdom of Saudi Arabia, e-mail: nftalic@ yahoo.com

\section{INTRODUCTION}

Air polishers have been available for use by dental professionals since the late 1970s. They are easy and efficient tools to remove extrinsic stains and plaque deposits from tooth surfaces. ${ }^{1,2}$ It is well documented that air polishing is more effective at removing stain and plaque deposits than conventional scaling and rubber cup polishing. Air polishing requires less time for stain removal and is less tiring for the dental practitioner. ${ }^{3}$ It has been shown that using air polishers on enamel surfaces is safe and does not cause any loss of enamel following the procedure. ${ }^{4,5}$ Air polishing may lead to gingival bleeding and abrasion. However, these effects are transient and have no clinical significance. $^{6,7}$

The first powder to be used with air polishers was sodium bicarbonate $\left(\mathrm{NaHCO}_{3}\right)$, which has a particle size of up to $250 \mu \mathrm{m} .{ }^{2}$ Manufacturers have recently developed other types of powders that have smaller diameter particles. These powders are safer, less abrasive, and have no salty taste. These powders include glycine (naturally occurring amino acid), calcium sodium phosphosilicate (bioactive glass), calcium carbonate, and aluminum trihydoxide. ${ }^{4}$

Previous studies have shown that air polishing is the most effective and efficient method for plaque removal around orthodontic brackets and arch wires ${ }^{8-10}$ However, a recent study found that the use of sodium bicarbonate powder may lead to higher frictional resistance with metal and ceramic brackets in vitro. ${ }^{11}$ A comparison of the in vitro effect of sodium bicarbonate and glycine powders showed that both powders had an effect on orthodontic metal brackets and wires, and there was no significant difference between the two powders. ${ }^{12}$ Air polishing increases the frictional resistance by $2.5 \%$ on average. ${ }^{12}$ These in vitro studies have certain limitations and do not represent the actual oral environment. To the best of our knowledge, no previous studies have examined the effect of air polishing on the surface topography of orthodontic arch wires.

The aim of this study was to examine the in vivo effect of air-powder polishing on the surface roughness and topography of aged orthodontic stainless steel arch wires that were qualitatively and quantitatively examined with a noncontact three-dimensional (3D) optical profilometer and scanning electron microscopy (SEM). 


\section{MATERIALS AND METHODS}

A total of 20 stainless steel arch wires (3M Unitek, Monrovia, California, USA) were used in this study. Ten wires (experimental) were aged and exposed to airpowder polishing, and 10 wires were used as received from the manufacturer, serving as control wires. The arch wires had a rectangular cross-section of $0.016 \times 0.022$ inches. The experimental group of wires were aged in the patient's oral cavity for an average of $5.3 \pm 2.7$ months. The arch wires were used during the finishing and detailing stage of treatment. They were not subjected to any sliding movements.

The patients' inclusion criteria were as follows: Patients included needed orthodontic treatment. At the time of retrieval of the wires, they required routine prophylaxis due to external plaque deposits and stains. All patients had upper and lower fixed orthodontic brackets $(0.018 \times 0.025$ inches; $3 \mathrm{M}$ Unitek). The procedure was explained to all patients included in the study, and a consent form was signed by each patient. Protective eye wear was placed on the patients' and clinicians' eyes. The procedure involved removing the ligature ties from the appliance. An air-powder polishing system (Prophy-jet; Dentsply/Cavitron, York, Pennsylvania, USA) was used for removal of stains and plaque deposits. The powder used in this study was sodium bicarbonate (Dentsply). Rapid, sweeping strokes were used at a distance of 4 to $5 \mathrm{~mm}$ from the tooth surface, with the tip directed at a $60^{\circ}$ angle to the teeth, as recommended by the manufacturer. Polishing for 10 seconds per tooth was usually adequate to remove stains and achieve prophylaxis. The total time for air-powder polishing for all six maxillary incisors and canines was 1 minute. Then, the wires were retrieved and placed in plastic bags marking the upper and lower surface of each arch wire.

The canine to canine segments of the experimental and control arch wires were subjected to surface topography analysis. The upper and lower surfaces of the experimental arch wires were evaluated. Only one surface of the control arch wires was examined. The surface topography measurements were performed using an optical noncontact surface profiling system (Bruker Contour GT-K; Bruker Nano GmbH, Berlin, Germany) based on white light interferometry to evaluate the 3D surface configuration and roughness of each arch wire surface. The machine was placed on a vibration isolation table in a super-silent room. Nine areas were randomly selected for each arch wire surface. The profilometer scanned area was approximately $1.25 \times 0.95 \mathrm{~mm}^{2}$ using an objective standard camera at $5 \times$ magnification. The data were processed using Vision 64 application software (Bruker Contour GT-K; Bruker GmbH, Berlin, Germany) to control the precision and measurements of the surface roughness parameters. The following three surface roughness parameters were determined: The arithmetic average height of the surface topography, also known as the average roughness $(\mathrm{Sa})$; the root mean square of the surface topography (Sq); and the 10-point height of the surface topography (Sz).

Following the profilometer analysis, a SEM (JSM 6360LV, JEOL Ltd, Tokyo, Japan) was used to evaluate the experimental and control arch wires. The SEM gives a qualitative morphological evaluation of the arch wire surfaces. The arch wires were prepared for scanning through coating with a gold foil using a fine coat machine (Ion Sputter JFC-1100, JEOL Ltd, Tokyo, Japan) for 3 minutes. The arch wires were then scanned by SEM using a high vacuum chamber pressure at $15 \mathrm{kV}$. Photomicrographs were taken of each experimental arch wire upper and lower surface at $200 \times$ magnification. Photomicrographs were taken for only one surface of the control arch wires at the same magnification.

Statistical analysis was performed using Statistical Package for the Social Sciences version 20 software (SPSS Inc., IBM, Chicago, Illinois, USA). The means, standard deviations (SDs), and minimum and maximum numbers were calculated for each surface roughness parameter. The upper and lower surfaces of the rectangular experimental arch wires were compared with the control using independent Student's t-test. The significance level was determined at $\mathrm{p}<0.05$. The reliability of the surface roughness results was examined by remeasuring three samples 1 week after the initial evaluation. Nine randomly selected areas on the arch wires were remeasured. There was no significant difference between the two measurements $(p=0.130)$.

\section{RESULTS}

The average roughness (Sa) of the experimental upper and lower surfaces exposed to air-powder polishing was significantly higher than the as-received control arch wires ( $p<0.01$; Table 1 and Graph 1). The root mean square of the surface topography $(\mathrm{Sq})$ was significantly higher on the upper and lower surfaces of the experimental arch wires compared with the control $(\mathrm{p}<0.05$ and $<0.01$ respectively; Table 2 and Graph 1). In addition, the 10-point height of the surface topography (Sz) of the

Table 1: Means and SDs of the average roughness (Sa) of the experimental and control arch wires

\begin{tabular}{llllll}
\hline Sa & Mean & SD & Max & Min & $p$-value \\
\hline Upper surface & 0.1514 & 0.0471 & 0.26 & 0.089 & $0.0091^{* *}$ \\
Lower surface & 0.1582 & 0.0414 & 0.256 & 0.117 & $0.0023^{\star *}$ \\
Control & 0.0961 & 0.0369 & 0.156 & 0.054 & \\
\hline${ }^{* *} p<0.01$ & & & & &
\end{tabular}




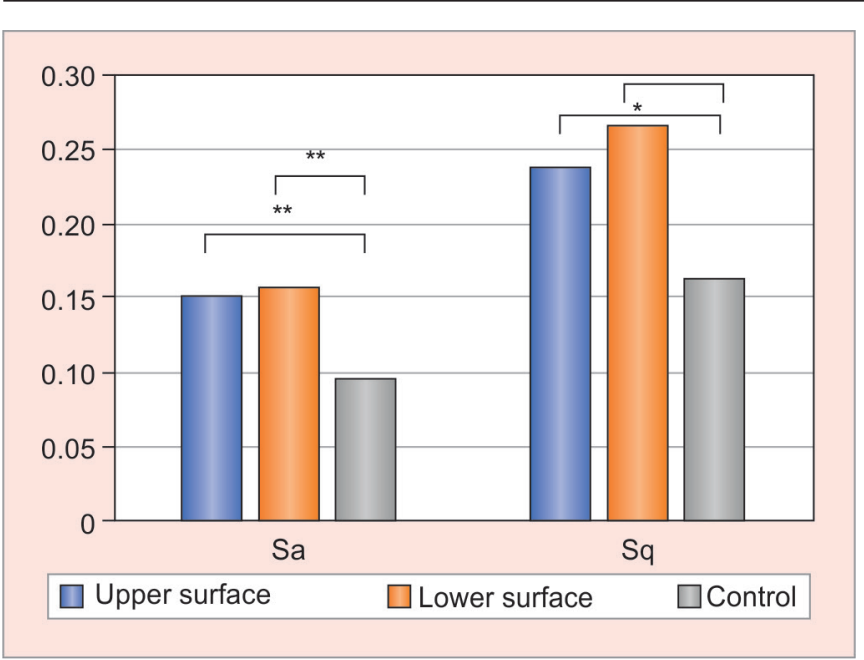

Graph 1: A comparison of the average roughness (Sa) and root mean square of the surface topography $(\mathrm{Sq})$ on the experimental upper and lower surfaces compared with the control. ${ }^{*} p<0.05$ and ${ }^{* *} p<0.01$

experimental arch wire upper and lower surfaces was significantly higher than the control arch wires $(\mathrm{p}<0.05$ and $<0.01$ respectively; Table 3). Evaluation of the SEM photomicrographs of the experimental and control arch wires indicated that the experimental arch wires were pitted and filled with surface impurities compared with the control arch wires, which appeared to be smooth without surface alterations (Fig. 1).

\section{DISCUSSION}

Fixed orthodontic therapy leads to increased biofilm buildup, which hinders oral hygiene practices. Performing oral prophylaxis can be challenging due to the nature and complexity of the appliances. Therefore, airpowder polishing is a good alternative in such situations. ${ }^{8}$ Debris buildup on the surface of wires and orthodontic brackets increases roughness. ${ }^{13,14}$ It has been shown that
Table 2: Means and SDs of the root mean square of surface topography $(\mathrm{Sq})$ of the experimental and control arch wires

\begin{tabular}{llllll}
\hline$S q$ & Mean & $S D$ & Max & Min & $p$-value \\
\hline Upper surface & 0.238 & 0.093 & 0.47 & 0.129 & $0.031^{*}$ \\
Lower surface & 0.2668 & 0.084 & 0.473 & 0.177 & $0.008^{* *}$ \\
Control & 0.1638 & 0.072 & 0.294 & 0.102 & \\
\hline${ }^{*} p<0.05,{ }^{* *} p<0.01$ & & & &
\end{tabular}

Table 3: Means and SDs of the 10-point height of surface topography $(\mathrm{Sz})$ of the experimental and control arch wires

\begin{tabular}{llllll}
\hline Sz & Mean & $S D$ & Max & Min & $p$-value \\
\hline Upper surface & 7.758 & 2.63 & 11.93 & 4.05 & $0.027^{*}$ \\
Lower surface & 9.058 & 2.09 & 11.99 & 5.85 & $0.0012^{* *}$ \\
Control & 4.457 & 3.18 & 10.66 & 1.72 & \\
\hline${ }^{*} p<0.05^{* *} p<0.01$ & & & & &
\end{tabular}

${ }^{*} p<0.05,{ }^{* *} p<0.01$

air-powder polishing is efficient and effective at removing plaque from orthodontically bracketed teeth. ${ }^{8,9}$ The procedure was well accepted by orthodontic patients, whereas they found the rubber cup and pumice method to be painful. ${ }^{1,9}$ In addition, air-powder polishing did not remove significant amounts of the resin composite used to bond orthodontic brackets. ${ }^{1,9,10}$

Air-powder polishing systems use a mix of air, water, and powder to deliver a controlled pressured stream of powder particles to tooth surfaces. It is well documented that air-powder polishing is an easy and effective tool used by hygienists, dentists, and dental specialists to remove plaque deposits and external stains from dental surfaces. ${ }^{3}$ It is more time efficient to use these systems to remove stains and plaque deposits from the tooth surfaces. In addition, it does not generate heat, which differs from other prophylaxis methods. ${ }^{8}$ There are several powder choices for these air-powder polishing systems. ${ }^{15-17}$ Sodium bicarbonate-based powders (NaHCO3) were initially used in air polishing. ${ }^{2} \mathrm{NaHCO} 3$ powders have
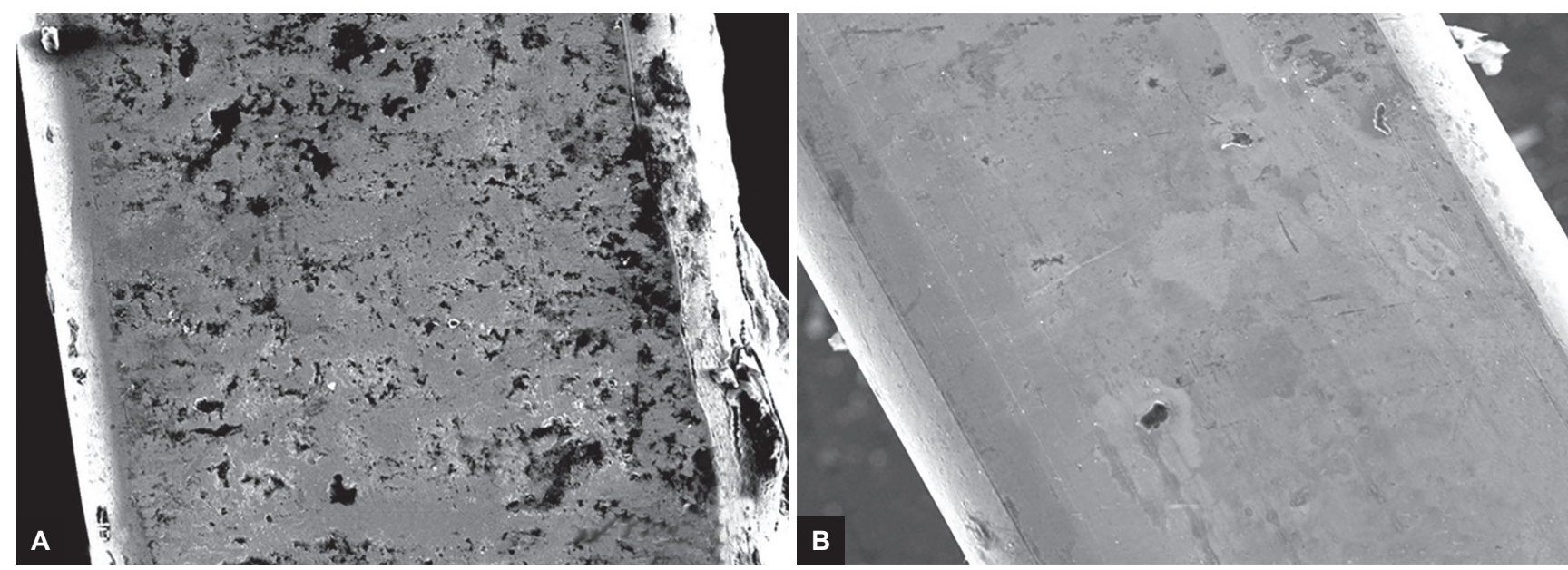

Figs 1A and B: (A) SEM photomicrograph of the experimental arch wire exposed to air-powder polishing at 200× magnification, which shows the surface roughness and pitting; and (B) SEM photomicrograph of the control arch wire at 200× magnification, which shows the smooth unaltered surface 
a particle size up to $250 \mu \mathrm{m} .{ }^{18}$ Studies showed that the supragingival use of $\mathrm{NaHCO}_{3}$ is safe and effective compared with conventional scaling and rubber cup polishing. ${ }^{2}$ Furthermore, it has been shown that both sodium bicarbonate and glycine-based powders had an in vitro effect on metal brackets and wires, and there was no significant difference between the two powders. ${ }^{12}$ Therefore, it was decided to examine the in vivo effect of sodium bicarbonate powder on the surface roughness and topography of orthodontic arch wires in this study.

Optical profilometry is a nondestructive, noninvasive alternative to contact profilometry that otherwise uses a stylus that measures surface roughness in straight lines, resulting in areas surrounding the line of measurement that are overlooked. ${ }^{19}$ The optical profilometer is equipped with a diamond tip. This tip has a radius of $5 \mu \mathrm{m}$ and is sensitive to vertical movements with an accuracy of s0.1 $\mu \mathrm{m} .{ }^{19}$ In addition, the nondestructive nature of the optical profilometer allowed for further analysis of the specimens using SEM in this study. The Sa, Sq, and $\mathrm{Sz}$ parameters are considered adequate in providing a complete topographic picture of the surfaces. ${ }^{20}$ All three parameters were significantly higher in the experimental wires as compared with the control. This shows that airpowder polishing does alter the surface topography and increase surface roughness, which may have an impact on the friction between the arch wires and brackets.

Friction is defined as the force that resists the movement of two objects sliding against each other. Frictional forces generated between orthodontic arch wires and brackets during sliding movements are a concern to every orthodontist because the force of friction impedes tooth movement. If the friction forces generated are high, treatment may be slow and unnecessarily long. ${ }^{19}$ Friction may lead to a loss in the applied orthodontic force in the range from $12 \%$ to more than $70 \% .{ }^{19,21}$ Furthermore, there is a relationship between the coefficient of friction and surface roughness. The higher the surface roughness, the higher the generated friction. ${ }^{19,21}$ Using air-powder polishing to remove deposits and stains during orthodontic treatment may alter the surface topography and friction coefficient. This study showed that air-powder polishing with sodium bicarbonate powder increases all three surface roughness parameters. The observations are in agreement with earlier reports that air polishing caused higher frictional resistance on both metal and ceramic brackets. ${ }^{11,12}$ In addition, surface alterations were observed in SEM photomicrographs. This is in agreement with previous studies showing that air-powder polishing my increase the friction generated between the wires and brackets by $2.5 \% .^{11,12}$ The arch wires filling the bracket slots will form a protective barrier against surface changes from air-polishers. Therefore, it is recommended to keep the arch wires in place during the procedure and then use a new arch wire after air-powder polishing. Another way to minimize the effect of air-polishers is to decrease the exposure time per tooth. Additionally, changing the angulation of exposure may minimize the surface changes associated with air-polishers, which merits further investigation. Future studies should examine the in vivo effect of air-powder polishing on other arch wire materials, such as nickel-titanium and titaniummolybdenum alloys. In addition, studying the effect of air-powder exposure time, changes in angulations, and developing less abrasive powders is warranted.

\section{CONCLUSION}

This is the first study to examine the effect of air-powder polishing on the surface roughness and topography in vivo. The air-powder polishing system using sodium bicarbonate powder may alter the surface topography and increase the surface roughness of aged orthodontic arch wires in vivo. The orthodontist may consider using a new arch wire after air-powder polishing to prevent the expected increase in friction, especially during the stages of treatment that involve sliding tooth movements.

\section{REFERENCES}

1. Gutmann ME. Air polishing: a comprehensive review of the literature. J Dent Hyg 1998 Summer;72(3):47-56.

2. Graumann SJ, Sensat ML, Stoltenberg JL. Air polishing: a review of current literature. J Dent Hyg 2013 Aug;87(4):173-180.

3. Christensen R. Oral prophylaxis: Prophy-jet. Clin Res Assoc Newsl 1981;5:1-5.

4. Boyde A. Airpolishing effects on enamel, dentine, cement and bone. Br Dent J 1984 Apr 21;156(8):287-291.

5. Gerbo LR, Lacefield WR, Barnes CM, Russell CM. Enamel roughness after air-powder polishing. Am J Dent 1993 Apr;6(2): 96-98.

6. Weaks LM, Lescher NB, Barnes CM, Holroyd SV. Clinical evaluation of the Prophy-Jet as an instrument for routine removal of tooth stain and plaque. J Periodontol 1984 Aug;55(8):486-488.

7. Kontturi-Narhi V, Markkanen S, Markkanen H. The gingival effects of dental airpolishing as evaluated by scanning electron microscopy. J Periodontol 1989 Jan;60(1):19-22.

8. Barnes CM, Russell CM, Gerbo LR, Wells BR, Barnes DW. Effects of an air-powder polishing system on orthodontically bracketed and banded teeth. Am J Orthod Dentofacial Orthop 1990 Jan;97(1):74-81.

9. Gerbo LR, Barnes CM, Leinfelder KF. Applications of the air-powder polisher in clinical orthodontics. Am J Orthod Dentofacial Orthop 1993 Jan;103(1):71-73.

10. Bennani V, Hwang L, Tawse-Smith A, Dias GJ, Cannon RD. Effect of air-polishing on titanium surfaces, biofilm removal, and biocompatibility: a pilot study. Biomed Res Int 2015;2015: 491047.

11. Parmagnani EA, Basting RT. Effect of sodium bicarbonate air abrasive polishing on attrition and surface micromorphology of ceramic and stainless steel brackets. Angle Orthod 2012 Mar;82(2):351-362. 
12. Wilmes B, Vali S, Drescher D. In-vitro study of surface changes in fixed orthodontic appliances following air polishing with Clinpro Prophy and Air-Flow. J Orofac Orthop 2009 Sep;70(5):371-384.

13. Marques IS, Araujo AM, Gurgel JA, Normando D. Debris, roughness and friction of stainless steel archwires following clinical use. Angle Orthod 2010 May;80(3):521-527.

14. Normando D, de Araújo AM, Marques Ida S, Barroso Tavares Dias CG, Miguel JA. Archwire cleaning after intraoral ageing: the effects on debris, roughness, and friction. Eur J Orthod 2013 Apr;35(2):223-229.

15. Aragón ML, Lima LS, Normando D. Air-powder polishing on self-ligating brackets after clinical use: effects on debris levels. Dental Press J Orthod 2016 Sep-Oct;21(5):90-94.

16. Camboni S, Donnet M. Tooth surface comparison after air polishing and rubber cup: a scanning electron microscopy study. J Clin Dent 2016 Mar;27(1):13-18.
17. Tastepe CS, Lin X, Donnet M, Wismeijer D, Liu Y. Parameters that improve cleaning efficiency of subgingival air polishing on titanium implant surfaces: an in vitro study. J Periodontol 2017 Apr;88(4):407-414.

18. Petersilka GJ. Subgingival air-polishing in the treatment of periodontal biofilm infections. Periodontol 20002011 Feb;55(1):124-142.

19. Bourauel C, Fries T, Drescher D, Plietsch R. Surface roughness of orthodontic wires via atomic force microscopy, laser specular reflectance, and profilometry. Eur J Orthod 1998 Feb;20(1):79-92.

20. Liu X, Ding P, Lin J. Effects of bracket design on critical contact angle. Angle Orthod 2013 Sep;83(5):877-884.

21. Nucera R, Lo Giudice A, Matarese G, Artemisia A, Bramanti E, Crupi P, Cordasco G. Analysis of the characteristics of slot design affecting resistance to sliding during active archwire configurations. Prog Orthod 2013 Oct 1;14:35. 